\title{
Confocal Holography: Improved Resolution through Object Scanning
}

\author{
R.A. McLeod, P. Jacquemin, S. Lai, and R.A. Herring \\ University of Victoria, Victoria, BC V8W 3P6 Canada
}

Confocal holography is a technique used to determine the internal index of refraction of materials in a non-invasive manner [1,2,3]. Briefly, the wavefront is split into two portions - an object and reference beam. The beams are then separately focused by telecentric optics. A sample is placed at the object beam focus and the reference beam passes through free space or a phantom. The two beams are then overlaid to form an interference pattern on a detector. Because the marginal and chief rays take different paths through the object, their contributions to the interference pattern are composed from different portions of the object. Through computer analysis, high resolution of the phase along the optical axis can be determined. Index of refraction can be further decomposed to find the internal temperature or composition of the object.

Previous examples of confocal holography have used an optical loop to allow the beam to be rastered through the object, based on a Dixon confocal microscope [4]. In order to develop a system with higher resolution, an object scanning system is being developed (Fig 1) in parallel with the original design. Due to the off-axis nature of the optics, the act of scanning the beam creates significant aberration, with a corresponding loss of resolution. In addition it increases the complexity of reconstructing the phase image. Keeping the laser beam stationary through the optical path and mechanically scanning the object through the beam profile has a number of advantages and disadvantages.

The primary advantages are improved optical performance and lower cost. The object scan design has fewer optical elements, so there is less optical aberration of the beam incident on the camera. The optical design is able to maintain a spot size of $7.5 \mu \mathrm{m} \mathrm{rms}$ at the object focus (Fig 2); the spot size and wavefront distortion are an order of magnitude better than the beam scanning system, while the object volume remains equivalent (a cubic centimeter). Approximately 120 fringes are formed on the detector, with over 36 pixels per fringe (Fig 3). Since the beam is stationary on the optics, the phase shift formed by the optics' surface irregularity are constant, and can be compensated for through the use of a single reference hologram. A redesign to achieve superior resolution can be performed by reducing the specified object volume.

With a smaller number of elements the phase noise from vibration of the microscope components is lessened. Compared to other holography methods, confocal holography has a large number of optical elements. As a result, structural vibration and acoustically coupled vibration from air conditioning has proven to be a major challenge. The benefit of a reduced part count is countered by the linear actuators used for scanning being directly coupled to the object. Hence it is more likely vibration generated by the actuators be transmitted directly to the object than in the case of the beam steering mechanism via galvanometers. Previous experimentation has shown that the system is more sensitive to microscale vibrations in the object than other optical elements. 
The greatest drawback to moving the object through the beam is that the motion of scanning may disturb it. For fluids experiments, this mandates that the system is only suitable for use at low Reynolds numbers where inertia is dominated by viscosity effects. The region where inertial effects may be neglected generally starts for channel diameters of $100 \mu \mathrm{m}$ or less. Thus most biological and microfluidic (e.g. fuel cell, lab-on-a-chip) applications can still be investigated without disturbing the object.

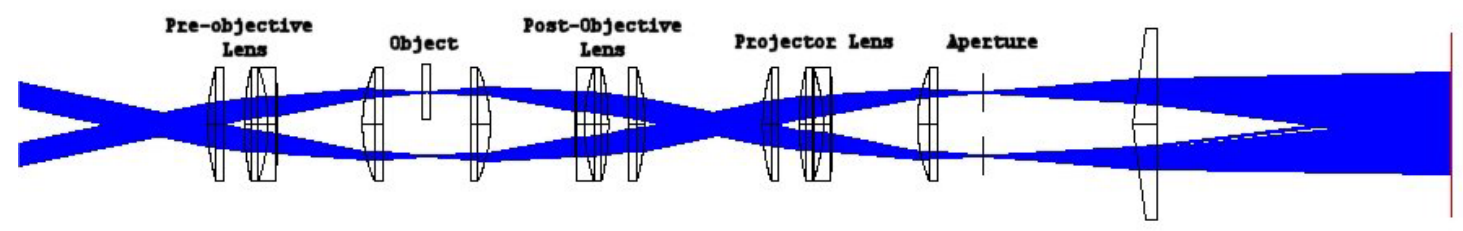

Figure 1: Optical Layout of Confocal Holography Microscope

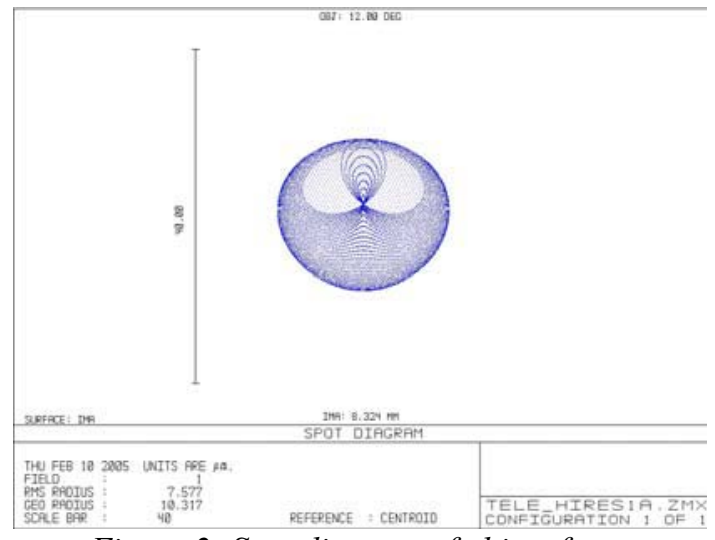

Figure 2: Spot diagram of object focus

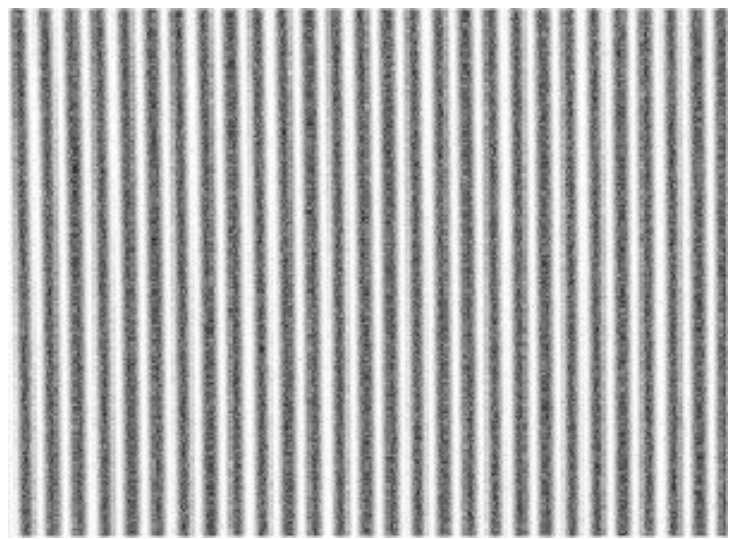

Figure 3: Sample fringe pattern

\section{References}

[1] R.A McLeod et al., Confocal Holography: A Tool for Non-Invasive Internal Measurement, Microscopy Today, 13 (January 2005) pp. 30-31.

[2] R.A. McLeod et al., A Viable Confocal Holography Micrscope and Method for HighLevel Measurements, Microscopy and Microanalysis 2004 (Savannah, GA) p. 1246. [3] R.A. Herring, Confocal scanning Laser holography, and an associated microscope: a proposal, Optik 105 (1997) pp. 65-68.

[4] A.E. Dixon, S. Damaskinos, M.R. Atkison: A scanning confocal microscope for transmission and reflection imaging. Nature 351 (1991) pp. 551-553. 